
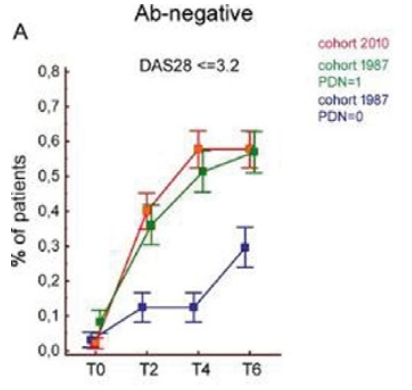

B

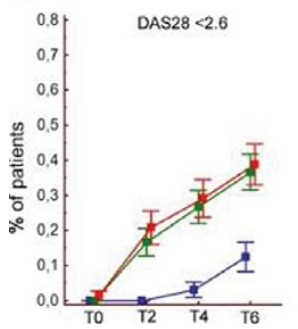

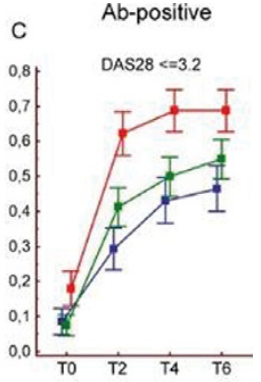

D

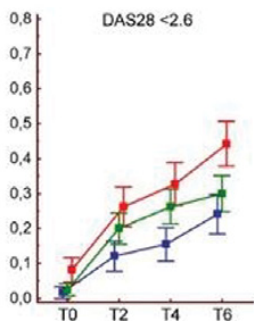

Disclosure of Interest: None declared

DOI: 10.1136/annrheumdis-2017-eular.5101

\title{
THU0100 IN EARLY INFLAMMATORY ARTHRITIS A LYMPHOID PATHOTYPE SIGNFICANTLY ASSOCIATES WITH REQUIREMENT FOR BIOLOGIC THERAPY AT 12 MONTHS FOLLOW UP: RESULTS FROM THE PATHOBIOLOGY OF EARLY ARTHRITIS COHORT (PEAC)
}

G. Lliso-Ribera ${ }^{1}$, F. Humby ${ }^{1}$, S. Kelly ${ }^{1}$, M. Bombardieri ${ }^{1}$, M. Lewis ${ }^{1}$, R. Hands ${ }^{1}$, V. Rocher ${ }^{1}$, F. Bene ${ }^{1}$, A. Nerviani ${ }^{1}$, C. Buckely ${ }^{2}$, P. Taylor ${ }^{3}$, I. McInnes ${ }^{4}$,

C. Pitzalis ${ }^{1}{ }^{1}$ Experimental Medicine and Rheumatology, Queen Mary University London, London; ${ }^{2}$ Centre for Translational Inflammation Research, University of Birmingham, Birmingham: ${ }^{3}$ Kennedy Institute of Rheumatology, University of Oxford, Oxford; ${ }^{4}$ Experimental Medicine and Rheumatology, University of Glasgow, Glasgow, United Kingdom

Background: Early aggressive treatment in RA equates to better long term outcomes, however targeting aggressive therapies including biologics to patients with the worse prognosis is critical to deliver acceptable risk/benefit ratios and health economic improvements. Such an approach requires prognostic biomarkers, whether the well recognised heterogeneity in synovial pathobiology in early RA translates to specific disease outcomes is currently unknown.

Objectives: The aim of this study was to investigate whether in a treatment naïve early inflammatory arthritis cohort, baseline synovial pathotype significantly associates with disease outcome at 12 months.

Methods: 166 consecutive DMARD naïve patients recruited as part of PEAC at Barts Health NHS Trust with synovial tissue suitable for analysis were included. At baseline patients were classified as RA (2010 ACR/EULAR criteria) or undifferentiated (UA). All patients underwent a baseline synovial biopsy of a clinically active joint along with collection of demographic data. Patients were subsequently treated with DMARD +/- steroid therapy with aim for low disease activity (DAS <3.3). At 6 month follow up patients were escalated to biologic therapy if fulfilling UK NICE guidelines. At 12 months patients were classified as: (i) no treatment, (ii) DMARDs, and (iii) Biologic +/- DMARDs. Sequentially cut sections of baseline synovial biopsies underwent immunohistochemical staining and semi-quantitative scoring (0-4) to determine the degree of CD20+Bcells, CD3+T cells, CD68+ lining (I) and sublining (sl) macrophage and CD138+ plasma cell infiltration. Sections were categorised into 3 pathotypes, (i) Fibroid: (CD68 $\mathrm{SL}<2$ and or CD3, CD20, CD138<1), (ii) Myeloid: (CD68SL $>2, C D 20<1$ and or CD3 $>1$ ) and (iii) Lymphoid: (grade 2-3 CD20+ aggregates, CD20>2).

Results: $79 \%$ were classified as RA and $21 \%$ as UA. Mean disease duration was 9.27 months. 92\% (153/166) patients had follow-up at 12 months. $29 \%(44 / 153)$ of patients were classified as fibroid, $34 \%(52 / 166)$ as myeloid and $37 \%(57 / 166)$ as lymphoid. At baseline patients with a lymphoid pathotype had a significantly higher CRP and DAS28 and were significantly more likely to be sero positive for for RF and ACPA $(p<0.05)$, suggesting that a lymphoid pathotype is associated with higher levels of disease activity. At 12 months follow up a significantly higher proportion of patients classified as lymphoid vs myeloid or fibroid (58\% vs $21 \%$ vs $21 \%$ ) required biologic therapy.

Conclusions: Results demonstrate that in an early inflammatory arthritis cohort

\begin{tabular}{lcccr}
\hline N=153 & Fibroid (44) & Myeloid (52) & Lymphoid (57) & p-value \\
\hline No treatment (14) n (\%) & $6(42 \%)$ & $6(42 \%)$ & $2(14 \%)$ & $<0.05^{*}$ \\
DMARD (101) n (\%) & $30(29 \%)$ & $38(37 \%)$ & $33(32 \%)$ & \\
Biologic +/- DMARD (38) n (\%) & $8(21 \%)$ & $8(21 \%)$ & $22(58 \%)$ & \\
\hline
\end{tabular}

a lymphoid pathotype significantly associates with higher disease activity at baseline, sero positivity for RF and ACPA and a requirement for more aggressive therapy at 12 month. This supports a direct role for synovial lymphoid structures in disease pathogenesis and suggests a role as a prognostic biomarker facilitating early stratification of aggressive therapeutic intervention

Disclosure of Interest: None declared

DOI: 10.1136/annrheumdis-2017-eular.6042

\section{THU0101 CARDIOVASCULAR MAGNETIC RESONANCE IMAGING CHARACTERISATION OF CARDIOVASCULAR ABNORMALITIES IN INDIVIDUALS AT RISK OF DEVELOPING RHEUMATOID ARTHRITIS}

G.J. Fent ${ }^{1}$, L. Hunt ${ }^{2,3}$, K.S. Mankia ${ }^{4,5}$, B. Erhayiem ${ }^{1}$, J.R. Foley ${ }^{1}$, P. Garg ${ }^{1}$, P.P. Swoboda ${ }^{1}$, J. Andrews ${ }^{4,5}$, J.P. Greenwood ${ }^{1}$, P. Emery ${ }^{4,5}$, S. Plein ${ }^{1}$, M.H. Buch ${ }^{1,5}{ }^{1}$ Leeds Institute of Cardiovascular and Metabolic Medicine, University of Leeds, UK; ${ }^{2}$ NIHR Leeds Musculoskeletal Medicine, University of Leeds, United Kingdom; ${ }^{3}$ Leeds Institute of Rheumatic and Musculoskeletal Medicine, University of Leeds, United Kingdom; ${ }^{4}$ Leeds Institute of Rheumatic and Musculoskeletal Medicine; ${ }^{5} \mathrm{NIHR}$ Leeds Musculoskeletal Medicine, University of Leeds, Leeds, United Kingdom

Background: Inflammation is the primary contributor to excess cardiovascular (CV) disease in rheumatoid arthritis (RA), with evidence of subclinical abnormalities observed even in treatment-naïve, early RA [1]. Preliminary reports suggest citrullinated proteins are present in atherosclerotic plaque [2]. It is unclear whether immunological changes of anti-citrullinated protein antibody (anti-CCP+) positive individuals "at risk" of developing RA are associated with CV abnormality. Objectives: To perform a pilot study to explore whether subclinical CV abnormalities are present in anti-CCP+ individuals at risk of developing RA.

Methods: Sixteen consecutive patients with non specific MSK symptoms but no synovitis, detectable anti-CCP antibody and 30 age-matched healthy controls (HC) underwent a multi-parametric 3.0T (Philips Achieva) cardiovascular magnetic resonance (CMR) study. Neither group had any history of CV disease. At-risk individuals were categorised as low and high absolute risk for RA development $(<50 \%$ and $\geq 50 \%$ respectively) according to a published risk model [3]. CMR post-processing was performed using CVI ${ }^{42}$ (Circle Cardiovascular Imaging, Canada).

Results: $\mathrm{HC}$ and at risk individuals were well matched for baseline characteristics (table 1). Aortic strain values (distensibility, strain and stiffness index $\beta$ ) were lower, indicating increased aortic stiffness, in at-risk individuals than $\mathrm{HC}$, numerically most pronounced those classified high risk (table 2). There were no differences in LV mass and function, late gadolinium enhancement, myocardial T1 (measure of myocardial composition) or LV S prime (Iongitudinal LV systolic function) (table 2).

Table 1. Baseine choracteristics

\begin{tabular}{|c|c|c|c|}
\hline & $\begin{array}{l}\text { All 'at risk' RA } \\
\text { potients } \\
(n=16)\end{array}$ & $\begin{array}{l}\text { Healtivy cont } \\
\text { trols } \\
(\text { nes } 30)\end{array}$ & D value \\
\hline Age (years) & 53211 & $50 \div 15$ & 0.59 \\
\hline Males & $4 / 16(25 \%)$ & $10 / 30(33 \%)$ & 0.57 \\
\hline Systolic BP (mmig) & $121: 17$ & $120: 13$ & 0.82 \\
\hline Dastolic BP (mentig) & $64=11$ & $64: 11$ & 0.81 \\
\hline $8 \mathrm{Mn}\left(\mathrm{kg} / \mathrm{m}^{2}\right)$ & $28 \div 5$ & $27 \pm 6$ & 0,47 \\
\hline Cabetes & $2 / 16$ & $0 / 30$ & 0.05 \\
\hline Active smoker & $1 / 16(6 \mathrm{~N})$ & $3 / 30(10 \mathrm{~N})$ & 0.68 \\
\hline Exesmoker & $11 / 16(69 \%)$ & $5 / 30(17 \%)$ & 0.001 \\
\hline Mean absolute pre- & $45 \mathrm{~K}$ & - & - \\
\hline
\end{tabular}

Table 2. Results able

\begin{tabular}{|c|c|c|c|c|c|}
\hline & $\begin{array}{l}\text { All 'at risk' RA } \\
\text { individuals } \\
(0=16)\end{array}$ & $\begin{array}{l}\text { LOW 'at risk' RA } \\
\text { individuals } \\
(n=10)\end{array}$ & \begin{tabular}{|l|} 
High 'at risK' RA \\
individuals \\
(n=6)
\end{tabular} & $\begin{array}{l}\text { Hesilthy } \\
\text { controls } \\
(n=30)\end{array}$ & p value \\
\hline $\begin{array}{l}\text { Acrocic Distersibility } \\
\left(10^{-1} \mathrm{~mm} H \text { ig) }\right.\end{array}$ & $3.6 \pm 1.4$ & $4.1 \pm 1.5$ & $2.8 \pm 0.5$ & $4.7 \div 2.0$ & 0.05 \\
\hline Aortic strain & $0.20: 0.06$ & $0.21 \div 0.07$ & $0.18 \div 0.03$ & $0.25=0.09$ & 0.03 \\
\hline $\begin{array}{l}\text { Acrtic stiffness index. } \\
B\end{array}$ & $3.5 \pm 1.0$ & $3.2 \pm 1.0$ & $4,040.8$ & $2.7 \pm 0.8$ & 0.007 \\
\hline Myocardial native $\mathrm{T1}$ & $1215 \div 35$ & $1213 \div 36$ & $2218 \div 37$ & $1202 \div 36$ & 0.25 \\
\hline $\begin{array}{l}\text { Late Gadolinium en- } \\
\text { hancement }\end{array}$ & $1 / 16$ & $0 / 6$ & $1 / 10$ & $2 / 30$ & 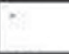 \\
\hline $\begin{array}{l}\text { IV ejection fraction } \\
\text { (s) }\end{array}$ & $62: 4$ & $62 \pm 5$ & $63: 3$ & $62 \pm 5$ & 0.94 \\
\hline $\begin{array}{l}\text { RV ejecion fraction } \\
\text { (S) }\end{array}$ & $55 \pm 6$ & $54 \pm 4$ & $58: 7$ & $54 \pm 6$ & 0.47 \\
\hline $\begin{array}{l}\text { Indexed LV mass ( } \mathrm{d} \text { ] } \\
\mathrm{m}^{2} \text { ) }\end{array}$ & $46 \div 10$ & $45 \pm 8$ & $49: 14$ & $49 \pm 8$ & 0.29 \\
\hline $\begin{array}{l}\text { Indexed tV volume } \\
\left(\mathrm{m} / \mathrm{m}^{2}\right)\end{array}$ & $83 \div 12$ & $81 \div 13$ & $87 \div 12$ & $80 \div 11$ & 0.34 \\
\hline tV S prime (s.1) & 1.1:0.1 & $1.2 \div 0.1$ & $1.0 \div 0.1$ & $1.1+0.1$ & 0.37 \\
\hline
\end{tabular}

